\title{
Short Hebrew International Physical Activity Questionnaire: Reliability and Validity
}

Authors' Contribution: A Study Design

B Data Collection

C Statistical Analysis

D Data Interpretation

E Manuscript Preparation

F Literature Search

G Funds Collection

\author{
Eyal Weissblueth \\ Ohalo Academic College of Education, Qatzrin, Israel \\ Department of Physical Education
}

\section{abstract}

Background

Material/Methods

Results

Conclusions

Key words

The International Physical Activity Questionnaire (IPAQ) aims to measure physical activity consistency throughout the world. It has been translated into many languages and its validity has been examined worldwide. However, the IPAQ has not been translated into Hebrew and the psychometric properties of the Hebrew version have yet to be evaluated.

The current study assessed the test-retest reliability of the short IPAQ in Hebrew (IPAQ-S-H) and its validity among college students. Intra-class correlation coefficients were computed to assess reliability, and survey responses were compared to $\mathrm{VO}_{2}$ max to determine concurrent validity.

The IPAQ-S-H's test-retest reliability was primarily high, particularly for total physical activity $(\mathrm{ICC}=0.90 ; 95 \% \mathrm{Cl}=0.87-0.93)$. While agreement for the physical activity components of the survey was higher (ICCs: 0.58-0.79), agreement for sitting time was lower (ICC $=0.39 ; 95 \%=0.25-0.52$ ). When comparing the survey scores to $\mathrm{VO}_{2}$ max, total physical activity and vigorous intensity activity were significantly correlated with maximal oxygen uptake (Pearson's $r=0.43$ and 0.40 , respectively), but not with other survey components.

The IPAQ-S-H primarily exhibits moderate reliability and validity, which are comparable psychometric properties to other IPAQ validation studies. Further validation is warranted among more representative samples utilizing objective monitoring to determine criterion validity.

physical activity, reproducibility of results, questionnaire

\section{article details}

Article statistics

Full-text PDF:

Copyright

Indexation:

Funding:

Competing interests: Corresponding author:

Open Access License:
Word count: 3064; Tables: 3; Figures: 0; References: 43

Received: September 2014; Accepted: February 2015; Published: March 2015

http://www.balticsportscience.com

(๑) Gdansk University of Physical Education and Sport, Poland

Celdes, CNKI Scholar (China National Knowledge Infrastructure), CNPIEC, De Gruyter - IBR (International Bibliography of Reviews of Scholarly Literature in the Humanities and Social Sciences), De Gruyter - IBZ (International Bibliography of Periodical Literature in the Humanities and Social Sciences), DOAJ, EBSCO - Central \& Eastern European Academic Source, EBSCO - SPORTDiscus, EBSCO Discovery Service, Google Scholar, Index Copernicus, J-Gate, Naviga (Softweco, Primo Central (ExLibris), ProQuest - Family Health, ProQuest - Health \& Medical Complete, ProQuest - Illustrata: Health Sciences, ProQuest - Nursing \& Allied Health Source, Summon (Serials Solutions/ProQuest, TDOne (TDNet), Ulrich's Periodicals Directory/ulrichsweb, WorldCat (OCLC)

An internal grant from Ohalo Academic College 2009. No external funding was received.

Author has declared that no competing interest exists.

Eyal Weissblueth, Ohalo Academic College of Education, Dept. of Physical Education, Qatzrin, 12900 Israel, Phone: +972-4-6825006, e-mail: eyalw@ohalo.ac.il

This is an open access article distributed under the terms of the Creative Commons Attribution-Non-commercial 4.0 International (http://creativecommons.org/licenses/by-nc/4.0/), which permits use, distribution, and reproduction in any medium, provided the original work is properly cited, the use is non-commercial and is otherwise in compliance with the license. 


\section{INTRODUCTION}

The effects of physical activity (PA) on health are well established. PA has been linked to the prevention of chronic diseases (e.g. type 2 diabetes, coronary heart disease, some cancers) and premature mortality [1-9]. For example, prospective observational studies and randomized controlled trials (e.g. Diabetes Prevention Program) have shown that moderate to vigorous intensity physical activity (along with a healthful diet) can significantly decrease the risk of diabetes incidence, even among pre-diabetics [10, 11].

Yet, despite these health benefits, in Israel only $32 \%$ of adults are sufficiently physically active in comparison to $51.7 \%$ of US adults $[12,13]$. Recommended physical activity for chronic disease prevention involves engaging in at least 150 of moderate or 75 minutes of vigorous intensity aerobic physical activity (or a combination of both), with additional health benefits achieved through higher amounts of activity (i.e. dose-response effect) [9].

A prerequisite for measuring (and subsequently increasing) physical activity on the population level necessitates consistent and standardized surveillance of activity levels. In the past two decades an international group of scientist has developed a standardized survey, the International Physical Activity Questionnaire (IPAQ) [14], to measure physical activity worldwide; its reliability and validity has been examined [15-19] in numerous countries worldwide [20-24], yet not in Israel [25-29]. Consequently, the current study was undertaken to assess the reliability and validity of the Hebrew version of the IPAQ. While versions of the IPAQ exist in Arabic (the prevalent language in the Middle East(30)), to our knowledge the IPAQ version in Hebrew (the prevalent language in Israel) has yet to be examined.

\section{MATERIAL AND METHODS}

The current study examined the test-retest reliability and construct validity of the short IPAQ in Hebrew among a sample of college physical education students in Northern Israel (March to May 2010). Prior to beginning the study, approval was obtained from the College Institutional Review Board, and participants provided written informed consent. A total of 214 undergraduate college students attending the Academic College of Education who majored in physical education were approached. Of these, 145 completed the entire questionnaire twice (test-retest reliability), and a sub-sample $(\mathrm{n}=47) \mathrm{com}$ pleted a maximal exercise test $\left(\mathrm{VO}_{2} \max \right)$ to determine construct validity. All participants consented to participate in the study, were aged $\geq 18$ years, had a medical health and fitness physician statement (required by Israeli law) that included no restriction on regular physical activity. In addition, prior to the study onset participants provided a specific consent statement concerning participation in the $\mathrm{VO}_{2}$ max test conducted on a treadmill.

The short, self-administered, version of the IPAQ (IPAQ-S), which measures total physical activity, was translated into Hebrew (IPAQ-S-H) by the study investigators while adhering to the recommended protocol in the IPAQ-S manual for translating self-reported PA into PA-related Energy Expenditure, as expressed in Metabolic Equivalent units (MET) [31]. Participants' responses to the questionnaire were scored based on scoring protocol, described elaborately elsewhere [32]. Briefly, the continuous score was used where all scores were 
expressed in MET-minutes/week. For the calculations of the three continuous scores for each of the PA types, the following was used: type of activity in MET-minutes/week = MET score of the activity multiplied by the minutes performed. Total PA MET-minutes/week was the sum of all three types of activities in MET-minutes/week. All METs were continuous.

Cardiorespiratory fitness was measured via $\mathrm{VO}_{2} \max \left(\mathrm{ml} \cdot \mathrm{kg}^{-1} \cdot \mathrm{min}^{-1}\right)$ testing based on the Bruce Protocol $[33,34]$. Briefly, the treadmill test was initiated with a speed equal to $2.8 \mathrm{~km}$ /hour with no slope. The slope was then increased by $3 \%$ every three minutes up to $24 \%$ (stage 8 ). The test required participants to exercise at an increasing workload until volatile exhaustion, requiring substantial physical effort; i.e., each participant was encouraged to continue to the best of their ability particularly during the latter stages of the test. A Polar heart rate monitor S610i ( $\pm 1 \%$ measurement accuracy) was used to measure the heart rate for the duration of the test. Modified Balke formula [34] was used to calculate maximal aerobic power using the last heart rate measurement at the final test stage.

Participants' age, gender, ethnicity, and perceived general health were assessed via a questionnaire. The body mass index was calculated using participants' objectively measured weight in kilograms divided by measured height in meters squared.

All participants completed the IPAQ-S-H questionnaire at baseline (Time 1: T1) and then four weeks later (Time 2: T2) to determine test-retest reliability. MET-minutes/week continuous values from baseline and from the second completion were compared using Intraclass Correlation Coefficients (ICC) and $95 \%$ confidence intervals for each survey item and for the total score. In addition, to determine construct validity, participants completed a $\mathrm{VO}_{2} \max$ test two weeks after completing the questionnaire at baseline [35]. Spearman correlation coefficients were computed to estimate the association of survey responses with cardiorespiratory fitness as measured by $\mathrm{VO}_{2}$ max. SPSS, Inc., Chicago IL, version 17.0 was used for all statistical analyses and significance was set at 2 tailed, $\alpha=0.05$ or lower.

\section{RESULTS}

Study participants' mean age was 24.2 years $(\mathrm{SD}=3.7)$, slightly less than half were women (49\%) and Arabs (45.6\%). In addition, on average, individuals were of normal weight (mean $\mathrm{BMI}=22.6, \mathrm{SD}=2.7$ ), and $86.4 \%$ perceived their health as either very good or excellent.

Participants' total physical activity (MET-minutes/week) as well as physical activity by intensity level (walking, moderate, vigorous) and sitting time at T1 and T2 are presented in Table 1. Participants' average total physical activity well exceeded the Health and Human Services physical activity guidelines with a mean MET-minutes/week of 3,337 (SD = 3,043) for T1 and 3,440 (SD $=3,259)$ for $\mathrm{T} 2$.

Analysis for the test-retest reliability of the IPAQ-S-H survey components indicated a moderate to high agreement (ICC: 0.59-0.79), with the highest reliability observed for the total score (ICC $=0.90 ; 95 \%$ CI $=0.87-0.93$ ) (cf. Table 
2). In comparison, sitting time was the least reliable survey component (ICC $=0.399 ; 95 \% \mathrm{CI}=0.253-0.528$ ). Furthermore, the correlation between survey components and cardiorespiratory fitness (to determine validity) is depicted in Table 3. Total physical activity was significantly and positively correlated with cardiorespiratory fitness (Pearson's $r=0.43 ; \mathrm{p}<0.01$ ). When examining the survey components, results reveal that the sitting, walking and moderate intensity physical activity scores were not correlated with cardiorespiratory fitness, whereas vigorous intensity activity was positively and significantly correlated with cardiorespiratory fitness levels (Pearson's $r=0.40 ; p<0.01$ ).

Table 1. Mean and standard deviation of responses to the IPAQ-S-H components and total score for Time 1 (T1) and Time 2 (T2), $\mathrm{n}=145$

\begin{tabular}{llll}
\hline Survey Scores & $\mathrm{n}$ & Mean (SD) T1 & Mean (SD) T2 \\
\hline MET-minutes/week Vigorous activity & 145 & $228(221)$ & $218(222)$ \\
\hline MET-minutes/week Moderate activity & 145 & $134(206)$ & $169(206)$ \\
\hline MET-minutes/week Walking & 145 & $248(485)$ & $256(469)$ \\
\hline Sitting (minutes per day) & 145 & $293(213)$ & $331(228)$ \\
\hline Total MET-minutes/week calculated & 145 & $3175(2719)$ & $3265(2910)$ \\
\hline
\end{tabular}

Abbreviations: IPAQ-S-H - International Physical Activity Questionnaire-Short-Hebrew; $\mathrm{SD}=$ Standard Deviation; MET = Metabolic Equivalent

Table 2. Test-retest reliability of the IPAQ-S-H: components and total score, $n=145$

\begin{tabular}{lll} 
Questionnaire item & $\begin{array}{l}\mathrm{N}=145 \\
\text { ICC }\end{array}$ & $95 \% \mathrm{Cl}$ \\
\hline MET-minutes/week Vigorous activity & $0.794^{*}$ & $0.726-0.847$ \\
\hline MET-minutes/week Moderate activity & $0.586^{*}$ & $0.469-0.683$ \\
\hline MET-minutes/week Walking & $0.734^{*}$ & $0.650-0.801$ \\
\hline Sitting (minutes per day) & $0.399^{*}$ & $0.253-0.528$ \\
\hline Total MET-minutes/week calculated & $0.904 *$ & $0.870-0.930$ \\
\hline
\end{tabular}

Abbreviations. IPAQ-S-H - International Physical Activity Questionnaire-Short-Hebrew; ICC = Intraclass Correlation Coefficients. $\mathrm{Cl}=$ Confidence Interval; $\mathrm{MET}=$ Metabolic Equivalent

$*=p<0.01$

Table 3. Construct Validity of the IPAQ-S-H: correlation of survey responses to $\mathrm{VO}_{2} \max , \mathrm{n}=47$

\begin{tabular}{lll}
\hline Survey Scores & Pearson's $r$ & $95 \% \mathrm{Cl}$ \\
\hline MET-minutes/week Vigorous activity & $0.403 *$ & $0.131-0.619$ \\
\hline MET-minutes/week Moderate activity & 0.221 & $-0.071-0.478$ \\
\hline MET-minutes/week Walking & 0.030 & $-0.259-0.314$ \\
\hline Sitting (minutes per day) & -0.063 & $-0.344-0.228$ \\
\hline Total MET-minutes/week calculated & $0.430 *$ & $0.163-0.638$ \\
\hline
\end{tabular}

Abbreviations. IPAQ-S-H - International Physical Activity Questionnaire-Short-Hebrew; MET = Metabolic Equivalent; $\mathrm{VO}_{2} \max =$ maximal oxygen uptake

$*=p<0.01$ 


\section{DISCUSSION}

The present study aimed to establish the test-retest reliability and construct validity of the IPAQ-S-H. To our knowledge, this is the first study to translate the IPAQ into Hebrew based on the recommended protocol [36] and to subsequently evaluate its psychometric properties. Study findings indicate that the IPAQ-S-H total score exhibited high reliability for all physical activity survey components. However, sedentary behavior, measured via one survey question pertaining to daily sitting time exhibited markedly lower reliability. This is in accord with previous [37] findings that sedentary behavior is not synonymous with lack of physical activity. When examining the validity of the survey, a higher total survey score (indicative of higher physical activity levels) was moderately correlated with increased cardiorespiratory fitness levels. When examining the survey components, vigorous intensity physical activity was associated with cardiorespiratory fitness, but not the moderate, walking or sitting scores.

Current findings emphasize that the IPAQ-S-H primarily exhibits similar reliability traits to the 12-country IPAQ validation study [25]. Thus, in the current study the coefficient for total physical activity equaled 0.90 , whereas in the 12 -country study the coefficient ranged from 0.32 to 0.88 (depending on the country). However, the reliability of the sitting time question was primarily lower than the 12-country study (current study: 0.39; 12-country study: 0.35-0.95). Further, when examining the validity of the IPAQ-S-H the correlation between the total physical activity and cardiorespiratory fitness was moderate (correlation coefficient of 0.43 ), which is similar to the correlation coefficients of the 12-country study (0.02-0.57; depending on the country). Caution should be taken, however, when comparing the validity of the current study to the 12-country study since physical activity was compared to accelerometer estimates and not cardiorespiratory fitness (as in the present study). Other validation studies [38, 39] comparing IPAQ's total physical activity to cardiorespiratory fitness found similar or lower correlation coefficients with VO2max than the present study. While cardiorespiratory fitness is an objective and direct consequence of physical activity, fitness is also impacted by genetic factors and weight status [40]. Thus, further validation of the IPAQ-S-H is warranted comparing the survey to objective monitoring of physical activity (e.g. accelerometry).

The current study has strengths and limitations. A notable strength stems from the fact that this is the first study to validate the IPAQ in the Hebrew language. Thus, once the Hebrew version of IPAQ is applied and utilized in Israel, this will enable international and consistent comparison of physical activity levels with other countries in the region and throughout the world. However, the current study offers an initial validation attempt examining a unique sample of college students majoring in physical education, who are not a representative sample of the general population in Israel. Furthermore, several studies [41, 42] have shown over-reporting PA that needs to be taken into consideration when interpreting PA questionnaire results. Hence further validation of the IPAQ-S-H is required among more representative samples in Israel. In addition, the study validated the short, self-administered, version of the IPAQ only; thus, validation of the telephone and long IPAQ in Hebrew are suggested. Finally, while consistent with other studies [43], the validation component of the study consisted of a subsample of participants rather than the larger sample utilized to examine the reliability of the survey. 


\section{CONCLUSION}

This study offers the first Hebrew version of the IPAQ, which exhibits moderate reliability and validity, which is comparable to other validation studies of the IPAQ in the world. The IPAQ-S-H could be utilized in Israel to assess physical activity, which will enable consistent comparison both within Israel and abroad. Further validation of the IPAQ-S-H is warranted in more representative samples while utilizing objective monitoring to determine criterion validity.

\section{REFERENCES}

[1] Kohl HWI. Physical activity and cardiovascular disease: evidence for a dose response. Med Sci Sports Exerc. 2001;33(6):s472-s483. [Available from: http://journals.lww.com/acsm-msse/Fulltext/2001/06001/ Physical_activity_and_cardiovascular_disease_.17.aspx]

[2] Morris J, Chave S, Adam C, Sirey C, Epstein L, Sheehan D. Vigorous exercise in leisure-time and the incidence of coronary heart-disease. Lancet. 1973;301(7799):333-9. [Available from: http://linkinghub.elsevier. com/retrieve/pii/S0140673673901281]

[3] Morris J, Heady J, Raffle P, Roberts C, Parks J. Coronary heart disease and physical activity of work. Lancet. 1953;ii:1053-7.

[4] Paffenbarger RS, Blair SN, Lee I-M, Hyde RT. Measurement of physical activity to assess health effects in free-living populations. Med Sci Sports Exerc. 1993 Jan;25(1). [Available from: http://journals.lww.com/ acsm-msse/Fulltext/1993/01000/Measurement_of_physical_activity_to_assess_health.10.aspx]

[5] Paffenbarger RS, Wing AL, Hyde RT. Physical activity as an index of heart attack risk in college alumni. Am J Epidemiol. 1995;142(9):889-903. [Available from: http://aje.oxfordjournals.org/content/142/9/889.abstract]

[6] Slattery ML, Jacobs DR. Physical fitness and cardiovascular disease mortality: The US railroad study. Am J Epidemiol. 1988;127(3):571-80. [Available from: http://aje.oxfordjournals.org/content/127/3/571.abstract]

[7] Berlin JA, Colditz GA. A meta-analysis of physical activity in the prevention of coronary heart disease. Am J Epidemiol. 1990 Oct;132(4):612-28. [Available from: http://view.ncbi.nlm.nih.gov/pubmed/214494]

[8] Powell KE, Thompson PD, Caspersen CJ, Kendrick JS. Physical activity and the incidence of coronary heart disease. Annu Rev Public Health. 1987;8:253-87. [Available from: http://view.ncbi.nlm.nih.gov/pubmed/3555525]

[9] Physical Activity Guidelines. [cited 2014 May 25]. [Available from: http://www.health.gov/PAGuidelines/]

[10] Manson JE, Nathan DM, Krolewski AS, Stampfer MJ, Willett WC, Hennekens CH. A prospective study of exercise and incidence of diabetes among US male physicians. JAMA. 1992 Jul;268(1):63-7. [Availa-ble from: http://view.ncbi.nlm.nih.gov/pubmed/1608115]

[11] Manson JE, Rimm EB, Stampfer MJ, et al. Physical activity and incidence of non-insulin-dependent diabetes mellitus in women. Lancet. 1991 Sep;338(8770):774-8. [Available from: http://view.ncbi.nlm.nih.gov/ pubmed/1681160]

[12] Israel Center for Disease Control. Health Status in Israel. Israel Ministry of Health; 2010. [Available from: http://www.health.gov.il/PublicationsFiles/Health_Status_in_Israel2010.pdf]

[13] Centers for Disease Control and Prevention (CDC). Behavioral Risk Factor Surveillance System Survey Data. Atlanta, Georgia: U.S. Department of Health and Human Services, Centers for Disease Control and Prevention; 2003.

[14] Hallal PC, Andersen LB, Bull FC, Guthold R, Haskell W, Ekelund U. Global physical activity levels: sur-veillance progress, pitfalls, and prospects. Lancet. 2012 Jul 27;380(9838):247-57. [cited 2014 May 25] [Available from: http://www.sciencedirect.com/science/article/pii/S0140673612606461]

[15] Deng HB, Macfarlane DJ, et al. Reliability and validity of the IPAQ-Chinese: the Guangzhou Biobank Cohort study. Med Sci Sports Exerc. 2008;40(2):303-7.

[16] Dinger MK, Behrens TK, Han JL. Validity and Reliability of the International Physical Activity Question-naire in College Students. Am J Health Educ. 2006 Dec 11;37(6):337-43.

[17] Hagströmer M, Oja P, Sjöström M. The International Physical Activity Questionnaire (IPAQ): a study of concurrent and construct validity. Public Health Nutr. 2006 Sep;9(6):755-62.

[18] Macfarlane DJ, Lee CCY, Ho EYK, Chan KL, Chan DTS. Reliability and validity of the Chinese version of IPAQ (short, last 7 days). J Sci Med Sport. 2007 Feb 1;10(1):45-51.

[19] Wolin KY, Heil DP, Askew S, Matthews CE, Bennett GG. Validation of the International Physical Activity Questionnaire-Short among Blacks. J Phys Act Health. 2008;5(5):746-60.

[20] Lachat CK, Verstraeten R, Khanh LNB, et al. Validity of two physical activity questionnaires (IPAQ and PAQA) for Vietnamese adolescents in rural and urban areas. Int J Behav Nutr Phys Act. 2008;5. [Availa-ble from: http://dx.doi.org/10.1186/1479-5868-5-37]

[21] Boon RM, Hamlin MJ, Steel GD, Ross JJ. Validation of the New Zealand Physical Activity Questionnaire (NZPAQ-LF) and the International Physical Activity Questionnaire (IPAQ-LF) with accelerometry. Br J Sports Med. 2010 Aug 1;44(10):741-6. 
[22] Maddison R, Ni Mhurchu C, Jiang Y, et al. International Physical Activity Questionnaire (IPAQ) and New Zealand Physical Activity Questionnaire (NZPAQ): a doubly labelled water validation. Int J Behav Nutr Phys Act. 2007;4:62.

[23] Ishikawa-Takata K, Tabata I, Sasaki S, et al. Physical activity level in healthy free-living Japanese esti-mated by doubly labelled water method and International Physical Activity Questionnaire. Eur J Clin Nutr. 2008;62(7):885-91.

[24] Egeland GM, Dénommé D, Lejeune P, Pereg D. Concurrent validity of the International Physical Activity Questionnaire (IPAQ) in an liyiyiu Aschii (Cree) community. Can J Public Health Rev Can Santé Publique. 2008 Aug 7;99(4):307-10.

[25] Craig CL, Marshall AL, Sjöström M, et al. International physical activity questionnaire: 12-country reliabil-ity and validity. Med Sci Sports Exerc. 2003;35(8):1381-95. [Available from: http://dx.doi.org/10.1249/01. MSS.0000078924.61453.FB]

[26] Vandelanotte C, de Bourdeaudhuij I, Philippaerts R, Sallis J. Reliability and Validity of a Computerized and Dutch Version of the International Physical Activity Questionnaire (IPAQ). J Phys Act Health. 2005;2(1):63. [Available from: http://search.ebscohost.com/login.aspx?direct=true\&db=s3h\&AN=15961249\&site=ehost-live]

[27] Booth M. Assessment of physical activity: an international perspective. Res Q Exerc Sport. 2000;71(2 Suppl):S114-20.

[28] Kurtze N, Rangul V, Hustvedt B-E. Reliability and validity of the international physical activity question-naire in the Nord-Trøndelag health study (HUNT) population of men. BMC Med Res Methodol. 2008;8:63. [Available from: http://dx.doi.org/10.1186/1471-2288-8-63]

[29] Lachat CK, Verstraeten R, Khanh LNB, et al. Validity of two physical activity questionnaires (IPAQ and PAQA) for Vietnamese adolescents in rural and urban areas. Int J Behav Nutr Phys Act. 2008;5:37. [Available from: http://dx.doi.org/10.1186/1479-5868-5-37]

[30] Downloadable questionnaires - International Physical Activity Questionnaire. [cited 2014 May 25] [Avail-able from: https://sites.google.com/site/theipaq/questionnaire_links]

[31] General info [Internet]. [cited 2013 Dec 6] [Available from: http://www.ipaq.ki.se/scoring.htm]

[32] Microsoft Word - IPAQ_final_nov05.doc - scoring.pdf. [cited 2013 Dec 6] [Available from: http://www.ipaq. ki.se/scoring.pdf]

[33] ACSM. ACSM's Primary Care Sports Medicine. McKeag D, Moeller JL, editors. Philadelphia: Lippincot Williams \& Wilkins; 2007.

[34] American College of Sports Medicine. Physical fitness testing. ACSM's Guidelines for Exercise Testing and Prescription. 1995, p. 49-78.

[35] Abramson JH. Survey Methods in Community Medicine: Epidemiological Studies, Programme Evalua-tion, Clinical Trials. 4 Sub edition. Edinburgh; New York: Churchill Livingstone; 1991, 285 p.

[36] Cultural adaptation - International Physical Activity Questionnaire. [cited 2014 May 26] [Available from: https://sites.google.com/site/theipaq/cultural-adaptation]

[37] Rosenberg DE, Bull FC, Marshall AL, Sallis JF, Bauman AE. Assessment of sedentary behavior with the International Physical Activity Questionnaire. J Phys Act Health. 2008;5 Suppl 1:S30-44.

[38] Mäder U, Martin BW, Schutz Y, Marti B. Validity of four short physical activity questionnaires in middle-aged persons. Med Sci Sports Exerc. 2006 Jul;38(7):1255-66.

[39] Rangul V, Holmen TL, Kurtze N, Cuypers K, Midthjell K. Reliability and validity of two frequently used self-administered physical activity questionnaires in adolescents. BMC Med Res Methodol. 2008;8.

[40] Kerem S, Kohl HW, Finley C, Barlow C, Gabriel K, Leonard D. Sedentary behavior, cardiorespiratory fitness, physical activity, and cardiometabolic risk in men: The Cooper Center Longitudinal Study. Mayo Clinic Proc. In Press.

[41] Fogelholm M, Malmberg J, Suni J, et al. International Physical Activity Questionnaire: Validity against fitness. Med Sci Sports Exerc. 2006;38(4):753-60.

[42] Rzewnicki R, Vanden Auweele Y, De Bourdeaudhuij I. Addressing overreporting on the International Physical Activity Questionnaire (IPAQ) telephone survey with a population sample. Public Health Nutr. 2003 May;6(3):299-305.

[43] Merom D, Delbaere K, Cumming R, et al. Incidental and planned exercise questionnaire for seniors: validity and responsiveness. Med Sci Sports Exerc. 2014 May;46(5):947-54. 